\title{
Influencia minoritaria sobre las opiniones frente al aborto y los anticonceptivos*
}

\section{Juan A. Pérez Gabriel Mugny}

Universidad de Ginebra

\section{INTRODUCCION}

Nuestros trabajos a lo largo de una docena de años sobre la influencia social y minoritaria en particular (Mugny, 1981) nos han llevado a estudiar los procesos de influencia en un contexto social complejo en el que la expresión de opiniones o actitudes están estrechamente relacionadas con la identificación y pertenencia a distintos grupos o categorías sociales. Se ha pasado así de la dimensión puramente intrapersonal y/o interpersonal, predominantes en los trabajos clásicos de la influencia (Mugny y Doise, 1980), a una dimensión más social.

Se trata así de integrar el modelo interaccionista formulado por Moscovici (1976), que ha hecho posible la conceptualización de los procesos de influencia minoritaria, y el modelo de la categorización (Tajfel, 1972, 1978) y su complementario de la diferenciación categorial (Doise, 1979) que abordan los procesos de relaciones entre grupos en términos de procesos cognitivos y afectivos de discriminación y diferenciación.

\subsection{El contexto social de la influencia minoritaria}

Según el modelo interaccionista el conflicto social debe ser considerado como base esencial de los procesos de la influencia en general y de la influencia de las minorias en particular. Es en la gestión de este conflicto como la minoria, desprovista en un principio de todo poder social reconocido (estatus, competencia, recompensa, etc.), puede obtener su impacto y difundir la innovación social. Pero, ¿cómo puede llegar a generar la minoría este conflicto? Son ya muchos los trabajos (véase Moscovici y Mugny, 1983) que han demostrado que el recurso de la minoría reside en su estilo o retórica de comportamiento, debiendo mostrar estabilidad, coherencia y firmeza a través del tiempo (consistencia diacrónica) y reflejando al mismo tiempo un consensus interno (consistencia sincrónica). Es así como la minoría rompe con la norma dominante y se define como una entidad diferenciada en el conjunto del campo social pudiendo ser percibida

* Fste estudio ha sido realizado en el marco del Fondo Nacional Suizo de Investigación Científica, N." 1.714 .0 .83 
como portadora de una alternativa innovadora.

En toda situación de influencia cabe distinguir de modo general al menos tres entidades bien diferenciadas (para más detalles véase Mugny, 1981): el poder, a menudo institucionalizado, que dicta $\mathrm{e}$ impone las normas a la población; en esta dualidad adquiere sentido la minoría caracterizada por su ruptura con el poder y por su intento de influenciar a la población. Naturalmente, en este contexto el poder intentará con todos los medios a su alcance impedir la difusión de la innovación, medios que van desde la represión brutal hasta mecanismos ideológicos mucho más sutiles como la psicologización (Mugny y Papastamou, 1980) o la denegación (Moscovici, Mugny y Pérez, en preparación). La población (por analogía con la población experimental) debe así tomar posición en una situación en la que todo acercamiento, como todo alejamiento, a la minoría resulta a su vez muy conflictual. La minoría deberá, pues, tener en cuenta esta serie de dinámicas psicosociales complejas. Si bien la minoría debe bloquear toda concesión al poder, no obstante, para obtener cierta influencia deberá negociar con la población con un estilo de negociación más flexible o más rígido (Mugny, 1981).

\subsection{Identificación e influencia social}

El modelo de la categorización (definido por la acentuación de diferencias entre estímulos que son percibidos como pertenecientes a categorias distintas $y$ por la acentuación de la similitud entre estímulos que son percibidos como pertenecientes a una misma categoría) elaborado por Tajfel $(1972,1978)$ y su extensión a los procesos de influencia (Turner, 1981) permiten abordar las relaciones de diferenciación y discriminación entre grupos en términos de mecanismos psicológicos cognitivos implicados en dichas relaciones. Se tendría así que la mera clasificación de los individuos en distintas categorias sociales puede gene- rar procesos de discriminación entre grupos (puede apreciarse, pues, la posible transposición al contexto intergrupal de las situaciones de influencia minoritaria: poder-población-minoria). Se postula además que uno de los procesos que están en la base de los comportamientos de diferenciación $y$ discriminación es que, mediante la competición social (sesgo evaluativo en favor del propio grupo), el individuo tiende a asegurar una identidad social positiva o, al menos, a evitar una identidad negativa (Turner, 1981).

Aplicando estos postulados a los procesos de influencia minoritaria (Mugny, 1981) se tendria que la minoria es percibida como una entidad bien diferenciada en el campo social y se le atribuyen una serie de características que se conocen de antemano o se elaboran en función de las situaciones. Toda respuesta de acercamiento a dicha minoría no implica meramente el grado de acuerdo con su posición, sino además indica el grado en que el individuo blanco de influencia está dispuesto a «identificarse» con tal minoría. Este proceso de identificación psicosocial supone tres aspectos complementarios:

a) un aspecto inferencial: la fuente de influencia será categorizada como una entidad social que será situada en el conjunto del campo social. Se verá cómo este proceso de categorización puede ser orientado por inducción experimental pudiendo examinarse así sus efectos directos sobre los comportamientos de discriminación y diferenciación (tanto a nivel evaluativo como comportamental) de los sujetos hacia la fuente minoritaria asi categorizada. Pero se puede también examinar en qué situaciones el sujeto tiende a elaborar por sí mismo una categorización del conjunto del campo social y de la fuente minoritaria y hasta qué punto dicha categorización es necesaria para que la minoría obtenga o no el efecto requerido.

Una de las predicciones que se deriva directamente de este aspecto es, como ha 
sido demostrado por Mugny y Papastamou (1982), que la influencia es mayor cuando el individuo es llevado a creer que tiene muchas categorias comunes con la fuente que cuando cree que tiene menos, en forma de sesgo sociocentrista.

b) un aspecto inductivo: en función de los diversos estereotipos existentes de antemano, el individuo conoce o elabora (en función de las distintas situaciones concretas) las características de cada categoría social distinguida en el campo social y por lo tanto también de la fuente minoritaria. En cada situación de influencia es de suma importancia conocer las caracteristicas de la fuente que resultan (por ejemplo un contexto normativo de originalidad difiere favorablemente de un contexto en el que sobresale la desviación, Mugny, Rilliert y Papastamou, 1981) puesto que de ellas dependerá en gran medida el grado de influencia obtenida por la fuente minoritaria.

c) un aspecto deductivo: el sujeto que adopta la posición específica de una categoría se autoatribuirá también las otras características (cfr. aspecto inductivo) de esa categoría que sobresale en dicha situación. Por otra parte, puesto que el individuo tiende a una búsqueda de identidad positiva, se supone que la aceptación o rechazo de la fuente minoritaria está relacionada con la valoración que el sujeto haya inducido de la fuente minoritaria. Se puede derivar la hipótesis de que cuando la imagen de la fuente minoritaria es muy negativa y cuando el individuo es llevado a creer que tiene muchas categorías comunes con la fuente, no se producirá el sesgo de sociocentrismo, sino el resentimiento de un conflicto de identificación, que se traducirá en mayor rechazo de la minoria en forma de una diferenciación o disimilación, tal como se ha demostrado (Lemaine, Lasch y Ricateau, 1971-72, Mugny, Kaiser y Papastamou, 1983).

\subsection{Hipótesis}

En el experimento que presentamos aqui se trata de examinar, por una parte, cómo elabora el sujeto la categorización de la fuente $y$, por otra, cómo reacciona cuando una categorización concreta le es dada. De igual modo, será la ocasión para poner a prueba el material de un nuevo paradigma experimental, referido a los juicios sociales sobre el tema del aborto. A pesar del carácter exploratio de este experimento, no obstante, se pueden avanzar diversas hipótesis de carácter general.

Así, en primer lugar, cabe suponer que la elaboración cognitiva de una categorización del campo social (relacionado con el tema del aborto, por ejemplo) no venga dada automáticamente por la simple lectura de la posición minoritaria, como es el caso en los paradigmas de influencia minoritaria basados sobre tareas perceptivas. En éstos la posibilidad de variabilidad de respuestas en la mayoria de las situaciones es binaria o bimodal (verdadero-falso, verde-azul, etcétera). La adopción de una de estas respuestas es así exclusiva y reenvía a una división categorial tajante (mayoría-minoría, normativo-contranormativo, normal-desviado, falso-correcto). Por el contrario, en los paradigmas fundados en los juicios sociales, en general, y tal como será el caso en nuestro estudio, las respuestas son plurimodales $y$, por lo tanto, cabe esperar categorizaciones múltiples y más complejas (lo que no excluye que en determinados casos unas categorizaciones estén más bipolarizadas, que otras, Paicheler, 1977). En lo referente al aborto el sujeto tendrá que contar con diversas categorias sociales (vgr.: la Iglesia, el gobierno, la mayoría, los jóvenes, las mujeres, etcétera). Una primera finalidad de este experimento será captar estas categorizaciones complejas.

Se tratará también de examinar si el sujeto concibe la alternativa minoritaria por la mera lectura de su posición o si, por el contrario, es necesario que el sujeto realice una actividad sociocognitiva estructurante sobre la representación e imagen de la minoría para llegar a situarla como una entidad diferenciada y alternativa en el campo social. Diferentes es- 
tudios han mostrado la relación entre la imagen de la fuente y la influencia obtenida por dicha fuente. A pesar de todo, a nuestro saber, no existe ningún trabajo que haya controlado el efecto mismo de la actividad de juzgar la fuente minoritaria. Paradójicamente se han manipulado los efectos de la imagen como obstáculo a la influencia social (cfr. la psicologización o la denegación, anteriormente citados), mientras que, al mismo tiempo, teóricamente se postula su rol positivo (véase Nemeth y Wachtler, 1974; Moscovici, 1976; Mugny, 1981). Nuestra hipótesis aqui es que la imagen constituye una red de lectura, aspecto inductivo, imprescindible para que se perciba claramente a la minoría como una entidad alternativa en el campo social (aspecto inferencial). Predecimos, pues, que la influencia obtenida por la minoría será mayor cuando los sujetos juzgan la fuente minoritaria sobre diversas características antes de expresar sus propias opiniones que cuando expresan dichas opiniones directamente después de haber leído la posición minoritaria. En el primer caso, ciertas de esas características (como la consistencia, en el sentido de reconocimiento de la minoría como alternativa en el campo social) podrían resaltar más en la interpretacion de la posición minoritaria, mientras en el segundo caso sólo sobresaldría la oposición de las posiciones respectivas.

Ya se ha mencionado la dificultad de predecir la categorización concreta de la fuente minoritaria que elaborará el sujeto en las situaciones de influencia social referidas a los juicios sociales. Es de suma importancia, pues, controlar en la medi$\mathrm{da}$ de lo posible la variabilidad de las eventuales categorizaciones de la fuente. Para ello se orientará en dos condiciones experimentales la categorización de la fuente minoritaria variando el grado de comunidad de pertenencia categorial entre el sujeto y la fuente. Así, los sujetos, todos de sexo femenino, recibirán en una condición el texto minoritario atribuido a una fuente con su misma pertenencia sexual - intragrupo - o de pertenencia sexual masculina -extragrupo-. Esto permitirá abordar el papel de los procesos de diferenciación y discriminación entre grupos (a nivel representacional y comportamental) en la influencia minoritaria.

Ahora bien, hay que. reconocer que a esta altura de los trabajos en este campo las hipótesis no son unívocas. Partiendo, en primer lugar, del modelo de la categorización o de la diferenciación categorial, la hipótesis más directa que se puede extraer para aplicarla a los procesos de influencia minoritaria es que una fuente categorizada como intragrupo obtendrá más influencia que la categorizada como extragrupo y que se producirá un sesgo valorativo más favorable al intragrupo que al extragrupo. Sin embargo, esta predicción así formulada presupone un "vacuum" social que no tiene en cuenta las caracteristicas estereotipadas que pueden aplicarse a cada grupo ya en función de la relación que pueda mantener con un objeto social dado ya en función de ciertas posiciones asimétricas que lo caractericen.

En efecto, si se tiene en cuenta que en función del contexto normativo al grupo minoritario de entrada se le pueden asociar características estereotipadas negativas (Mugny y Papastamou, en prensa) y, por otra parte, teniendo en cuenta que el sujeto tiende a preservar su identidad positiva (evitando, pues, toda identificación con entidades sociales portadoras de una identidad negativa), se puede también suponer que cuando se trata de una fuente minoritaria, pero intragrupo, la identidad de facto del sujeto puede estar más amenazada que cuando se trata de un extragrupo. Asi, en caso del intragrupo, la identidad del sujeto se sentiria más interpelada que en el caso del extragrupo, pudiendo suceder que intente crear una diferenciación intracategorial que se traduciría tanto por una disminución de la influencia como por una valoración más negativa de la fuente (cfr. Mugny, Kaiser y Papastamou, 1983).

Por otra parte, se ha demostrado (Pérez y Mugny, en prensa) que cuanto más 
representada esté en la fuente minoritaria la definición (prototípica) de una categoría y que además esta categoria tenga cierta comunidad de pertenencia con el sujeto, más aumenta el conflicto de identificación con la fuente minoritaria, por lo que la influencia, si no es nula, al menos tiende a desplazarse sobre una dimensión indirecta. En el experimento que se presentará aquí se podría suponer de igual modo que la actitud frente al aborto implica más directamente la identidad de la categoría sexual femenina que la masculina (Maass y cols., 1982). Por lo tanto la fuente minoritaria intragrupo acentuaría así también la cuestión de la identificación más que la fuente minoritaria extragrupo lo que podría traducirse en un mayor intento, tanto a nivel representacional como comportamental, del sujeto de la condición intragrupo por diferenciarse de la fuente minoritaria intragrupo.

Según la concepción de Nemeth y Wachtler (1974) y de Maass, Clark y Haberkorn (1982), finalmente, se puede predecir que una minoría ejercerá menos influencia cuando sea percibida como guiada por su interés propio ("self-interest»). Si nos atenemos a la lógica del párrafo precedente, se debería concluir que a la fuente minoritaria femenina (intragrupo) se le atribuirá más interés propio (luego menos influencia) que a la fuente minoritaria masculina (extragrupo). Ahora bien, en otra lógica, si el interés propio no se atribuye en función de la relación entre la actitud especifica de la fuente y el objeto social en cuestión (en este caso las mujeres serían consideradas como más directamente interesadas en la legalización del aborto), sino en función de la categorización de la fuente como extragrupo, como expresión de una discriminación y diferenciación, en este caso el sesgo de interés propio seria atribuido a la fuente masculina.

Frente a esta heterogeneidad y en la medida en que no conocemos la categorización efectuada por los sujetos, dejaremos al experimento (insistimos sobre su carácter exploratorio) la tarea decisoria.

\section{METODO}

\subsection{Los sujetos}

Un total de 203 sujetos, todos de sexo femenino, de una media de edad de 17 años aproximadamente, pertenecientes a un Instituto Femenino de Enseñanza Media de una ciudad de España, han participado en este experimento. Se utilizó como lugar de experimentación las aulas mismas en las que los sujetos habitualmente siguen sus clases. Todas las condiciones experimentales fueron repartidas aleatoriamente en cada uno de los grupos escolares. El experimento fue realizado en el mes de noviembre de 1983.

\subsection{Procedimiento y diseño experimental}

El objetivo de este experimento, que transcurrió en una sola fase, es abordar tres puntos: el impacto del mensaje en el que se defiende la legalización y gratuidad del aborto sobre la expresión de las opiniones de los sujetos referidas al aborto y a los anticonceptivos; el efecto de la representación del grupo minoritario en los procesos de influencia y el efecto sobre las opiniones de la acentuación de la comunidad de pertenencias categoriales entre el sujeto y la fuente.

Para medir el impacto del mensaje, en una condición los sujetos, después de haber contestado a un breve pretest, leían un texto favorable a la legalización y gratuidad del aborto atribuido a un grupo minoritario de jóvenes y contestaban a continuación, $y$ en este orden, a un cuestionario de opiniones, a un cuestionario de la representación del campo social y a un cuestionario de la imagen de la fuente (se llamará a esta condición fuente-opinión), que serán descritos más adelante. En otra condición los sujetos contestaban, en este orden, al cuestionario de opiniones, al cuestionario de la representación del campo social y al cuestionario de la imagen de la fuente mino- 
ritaria. En esta condición (que llamaremos condición control) los sujetos no leían el texto del grupo minoritario de jóvenes (para el cuestionario de la representación de la fuente se indicaba a los sujetos que debían responder imaginándose un grupo minoritario de jóvenes que estuviesen a favor del aborto). Estas dos condiciones sólo difieren, pues, por la presentación o no del texto minoritario, siendo idéntico el orden en el que los sujetos contestaron a los diversos cuestionarios.

El papel de la imagen de la fuente minoritaria fue controlado variando el orden en el que los sujetos debían contestar a los cuestionarios. Así, se creó una condición en la que los sujetos después de leer el texto atribuido a un grupo minoritario de jóvenes respondian al cuestionario de la representación de la fuente, terminando por el cuestionario de opiniones y el de la representación del campo social (llamaremos a esta condición fuente-imagen). Para examinar, pues, el papel de la imagen de la fuente sobre las opiniones de los sujetos, se comparará esta condición a la condición fuente-opinión, anteriormente descrita. En efecto, estas dos condiciones difieren únicamente en el orden de presentación del cuestionario de la representación de la fuente, ya antes del cuestionario de opiniones ya en último lugar; será posible así estudiar el papel mediacional de la imagen de la fuente sobre las opiniones de los sujetos frente al aborto y los anticonceptivos.

Para examinar el efecto sobre las opiniones del grado de comunidad de pertenencia categorial entre el propio sujeto y la fuente minoritaria, se crearon dos condiciones adicionales (el orden en el que estos sujetos realizan las diferentes tareas experimentales fue: pretest, lectura del texto del grupo minoritario, cuestionario de la representación de la fuente, cuestionario de opiniones y cuestionario de la representación del campo social): ęn un caso el texto minoritario fue atribuido a un grupo minoritario de jóvenes mujeres y en otro a un grupo minoritario de jóvenes bombres. Puesto que los suje- tos son todos de sexo femenino, en base a la identidad sexual de los sujetos, en el primer caso se trata de la misma pertenencia categorial (condición fuente intragrupo), no dándose en el segundo dicha comunidad de pertenencia categorial (condición fuente extragrupo). La comparación de estas dos condiciones permitirá abordar el efecto de la comunidad de pertenencias categoriales en los procesos de influencia.

\subsection{Material experimental}

\subsubsection{Pretest}

Con el fin de disponer de un indicador de la opinión general de los sujetos frente al aborto, los sujetos debían expresar, antes de toda inducción experimental (pretest), su grado de acuerdo o de desacuerdo a través de una escala de 7 puntos ( $1=$ totalmente en desacuerdo, $7=$ totalmente de acuerdo) a este primer cuestionario compuesto por los cinco items siguientes: "hay que legalizar el aborto»; "las menores de edad también deben poder abortar»; «legalizar el aborto es una exigencia democrática»; "el aborto tiene que ser gratuito para todo el mundo»; "siempre que una mujer ha quedado embarazada por violación hay que poder aplicarle el aborto".

Las respuestas a este primer cuestionario servirán de base para analizar al menos tres aspectos distintos. Obviamente, en primer lugar servirán para disponer de una apreciación global de la posición de la población experimental frente al aborto. Más importante aún, se podrá construir un indice del posible cambio de opiniones operado tras la fase experimental, puesto que los sujetos contestarán de nuevo a estos mismos cinco ítems después de haber leído el mensaje favorable al aborto (excepto en la condición control en la que este índice servirá para controlar el efecto de la mera presentación de los ítems); este índice de cambio de opiniones se obtendrá calculando la diferencia entre las respuestas dadas en el 
pretest y las dadas a esos mismos cinco items después de la fase experimental propiamente dicha. Y, por último, constituirá un medio de control para neutralizar las posibles diferencias entre condiciones experimentales debidas a los errores aleatorios que puedan ocurrir en la distribución al azar de los sujetos en diferentes condiciones experimentales.

\subsubsection{El texto}

Una de las fases claves de los experimentos de influencia social está constituida tanto por el contenido como por la forma retórica con la que se presenta la posición hacia la que se pretende influenciar al sujeto. En el experimento que se presenta aquí, en la fase de influencia propiamente dicha (salvo en la condición control), los sujetos leerán un texto atribuido a un grupo minoritario de jóvenes en el que se defiende una posición claramente a favor del aborto.

Este texto se estructura en torno a dos dimensiones: la legalización y la gratuidad del aborto. Cada una de estas dos dimensiones contiene a su vez dos reivindicaciones. Así, en la dimensión de la legalización se defiende que es de suma urgencia manifestarse públicamente exigiendo la legalización del aborto $\mathrm{y}$, por otra parte, que es necesario elaborar un estatuto juridico que legitime el recurso al aborto. El texto, titulado "por la legalización y la gratuidad del aborto», decia literalmente:

"Uno de los temas que necesita ser abordado en profundidad es el del aborto. Nuestro grupo minoritario de jóvenes ha tomado conciencia de ello desde hace tiempo, manifestando püblicamente la necesidad urgente de legalizar el aborto, es decir, darle el estatuto juridico tal como una verdadera democracia lo requiere.

Ahora bien, la legalización del aborto no es suficiente. En efecto, si con esta legalización toda la gente tendrá las mismas posibilidades legales de abortar, es evidente que no ocurre lo mismo desde el punto de vista económico. Una operación de aborto es costosa y no todas las clases sociales tienen las mismas posibilidades de acceder a él si cada uno tiene que asumir personalmente los costos de un aborto.»

En la segunda parte del texto se presentaban dos reivindicaciones referidas ambas a la dimensión de la gratuidad, en el sentido de una igualdad de clases. Por una parte, se defendia que el aborto debe ser totalmente gratuito $y$, por otra, que la Seguridad Social debe pagar todos los costos de un aborto. Textualmente se decía:

«Cuando nuestro grupo minoritario de jóvenes reivindica la legalización del aborto, exige además que sea gratuito para todo el mundo. Así, el aborto tiene que ser incluido en el seguro de enfermedad igual que cualquier otro riesgo, es decir, la Seguridad Social tiene que asumir automáticamente los costos de un aborto. Sólo asi todas las clases sociales, ricos y pobres, tendrán las mismas posibilidades de poder recurrir al aborto. Sólo así el aborto será verdaderamente democrático.»

El texto se terminaba reiterando las dos dimensiones centrales:

«En sintesis, nuestro grupo minoritario de jóvenes reivindica la legalización del aborto y que éste sea totalmente gratuito para toda la gente.»

Al igual que el contenido, también «la retórica» del texto se articula sobre diversas dimensiones: como puede apreciarse, las dos reivindicaciones centrales aparecen reiteradas veces (se encuentran hastà cinco referencias a cada dimensión en el texto) incidiendo así en una forma de coberencia interna. Por otra parte, expresiones como «nuestro grupo minoritario de jóvenes ha tomado conciencia de ello desde hace tiempo» y «cuando nuestro grupo minoritario de jóvenes reivindica" aluden al consensus intragrupo, dejando entrever una trayectoria consolidada en la manifestación de las reivindicaciones a través del tiempo; se notará 
que la retórica del texto minoritario reposa en efecto sobre la definición de la consistencia comportamental, a la vez diacrónica y sincrónica. Se puede notar por último la "firmeza" contenida en el estilo de ciertas expresiones (vgr.: «necesidad urgente", "reivindica», "exige además», «tiene que», "sólo así»).

\subsubsection{Cuestionario de la representacion de la fuente}

El interés de la medida de la representación del grupo minoritario que elaboran los sujetos ha sido ya probado a lo largo de un gran número de experimentos (cfr. Mugny, 1981). Ha sido así posible descifrar algunos procesos por los que atraviesa la influencia minoritaria. Siendo asi que dos condiciones experimentales pueden ser equivalentes en el grado expresado de aproximación con $\downarrow a$ fuente $y$, sin embargo, que se haya llegado a ese mismo resultado por procesos muy distintos y con distinta significación psicosocial. El empleo de este cuestionario to situamos completamente en esta linea.

Para la inclusión de los diferentes items en este cuestionario se han tenido en cuenta, por una parte, las dimensiones que en los diferentes estudios de influencia social (más particularmente minoritaria) se han revelado más pertinentes (para una revisión véase Mugny, 1981). Por otra, se han tenido también en cuenta las dimensiones más pertinentes en el tema del aborto.

Este cuestionario está constituido por 30 escalas bipolares de 7 puntos. Semejante a un diferenciador semántico, se organiza sobre varias dimensiones introducidas a priori. Se introdujo así la dimensión de la consistencia (vgr.: coherente-incoherente, seguro de sí mismo-no seguro de sí mismo), de la tolerancia (vgr.: tolerante-intolerante, transigenteintransigente, flexible-rígido), la de representatividad (vgr.: portavoz-no representa a nadie, convincente-charlatán), la de credibilidad (vgr.: digno de fe-no digno de $\mathrm{fe}$, competente-incompetente), la dimen- sión de moralidad (vgr.: moral-inmoral, egoísta-generoso) y la de autonomia (vgr.: partidista-autónomo, independiente-dependiente, individualista-sectarista). El resto de los items que componen este cuestionario son: humanitario-político, reaccionario-progresista, viejo-joven, maduro-inmaduro, mayoritario-minoritario, marginal-integrado, democrático-autoritario, feminista-antifeminista, realistairrealista, atrevido-no atrevido, críticoacrítico, abierto-lleno de prejuicios y objetivo-no objetivo.

\subsubsection{Cuestionario de opiniones}

Para la construcción de este cuestionario se han seguido dos fases sucesivas. La primera fase consistió en llevar a cabo un sondeo sobre la base de 48 items organizados sobre varias dimensiones. De èste cuestionario inicial fueron seleccionados finalmente 25 items basando la selección tanto en un criterio estadístico (alfa de Cronbach) como en el contenido no ambiguo de los ítems.

Este cuestionario final está compuesto de dos grandes dimensiones, una referida al aborto y otra a los anticontraceptivos, compuestas a su vez de una serie de subdimensiones. En la dimensión del aborto se tiene así que los ítems $6,9,11,13,21$, 24 y 25 (véase el cuestionario en el anexo) refieren los aspectos legales propiamente dichos del aborto. Otra serie de items versan sobre la igualdad social frente al aborto contenida en los ítems 3 , $4,5,7,8,15$ y 22 . Los items $1,2,17$ y 20 se refieren a la institucionalización del aborto. Como puede verse, el aspecto legal y la gratuidad del aborto constituyen dos ejes centrales de estos ítems y, como se recordará, son las reivindicaciones defendidas directamente en el texto.

La otra dimensión global, referida a los anticontraceptivos, contiene también diversos aspectos que pueden ser destacados: como alternativa al aborto (items 10 y 19), la publicidad de los anticontraceptivos (items 14 y 16) o la referida directamente a los anticontraceptivos en general (items 12, 18 y 23). 
Así pues, los ítems referidos al aborto están directamente relacionados con el texto que defiende la legalización y la gratuidad del aborto (cf. apartado 1.3.2); están dirigidos, pues, a captar de modo directo el impacto del mensaje. Consideramos que una respuesta de aceptación o rechazo de estos items constituye la reacción directa al texto por parte del sujeto, blanco de influencia. En el mismo orden, se considerará que estos items referidos al aborto "constituyen la dimensión directa de medida del impacto del mensaje $y$, puesto que la fuente se define a través de dicho mensaje, constituyen también una medida del grado en que los sujetos están dispuestos a aceptar a nivel directo (manifiesto) la fuente minoritaria.

Por el contrario, los items referidos a los anticonceptivos medirán el posible impacto del texto (referido únicamente al aborto) sobre esta dimensión de los anticonceptivos a la que la fuente minoritaria no ha hecho ninguna referencia y que sólo se puede inferir indirectamente la posición de la fuente minoritaria. Se considerará, pues, que los items sobre los anticonceptivos miden el impacto del texto sobre una dimensión que no le es directamente definicional, por lo que la consideraremos como dimensión indirecta.

Se tienen, pues, dos criterios sobre los que definir la dimensión directa e indirecta (dos dimensiones de suma importancia para la problemática que se tratará): no sólo por haber sido tratada directamente por la fuente en el texto que le fue atribuido, sino además por referirse a un contenido diferente, aunque sin duda con cierta relación (como lo muestra la correlación entre la dimensión del aborto $y$ la de los anticonceptivos: $r=0,60$, $\mathrm{p}<0,001$ ).

\subsubsection{Cuestionario de la representación del campo social}

Como ya se mencionó, uno de los objetivos importantes que nos proponemos con este nuevo paradigma experimental es situar los procesos de influencia social en un contexto social en el que la expre- sión de las opiniones está estrechamente relacionada con la identificación y pertenencia a distintos grupos o categorías sociales. De igual modo, hemos postulado que el grupo minoritario, para ejercer su influencia, tiene que ser percibido como una entidad social portadora de una alternativa bien diferenciada dentro del conjunto del campo social.

Asi pues, era necesario operacionalizar una medida que nos informase de la representación que los sujetos realizan del campo social y que al mismo tiempo llevase a los sujetos a «sortear» su propia posición entre el conjunto de entidades, que por definición se contrastan entre ellas diferencialmente. Es decir, se trata de medir las mismas opiniones del sujeto frente a las dos dimensiones introducidas en el cuestionario de opiniones, pero esta vez llevando al sujeto a que tenga presente directamente el conjunto del campo social. Asi, la expresión de su propia opinión significará visiblemente una identificación (proximidad) o una diferenciación (alejamiento) con las categorias sociales pertinentes y, por otra parte, se dispondrá de una mẹdida que indique en qué grado el grupo minoritario constituye la entidad alternativa por excelencia.

Por todo ello se construyó este cuestionario de la representación del campo social en el que los sujetos debían situar sobre una misma escala (esta vez de 11 puntos: $1=$ totalmente en contra y 11 $=$ totalmente a favor) la actitud que ellos creían que correspondía a diferentes entidades sociales frente a la legalización del aborto, la gratuidad del aborto, la publicidad del aborto, los anticonceptivos y la publicidad de los anticonceptivos.

Los sujetos comenzaban situando en primer lugar su propia posición en la escala (escribiendo "YO» en la casilla que les convenía mejor) y a continuación debían situar respectivamente para cada uno de los cinco ítems mencionados al grupo minoritario (a los sujetos de la condición control, al igual que para el 
cuestionario de la representación de la fuente, se les indicaba que situasen a un grupo minoritario imaginario que estuviese a favor de la legalización y gratuidad del aborto), al gobierno, a la mayoría, a la Iglesia, a los hombres, a las mujeres y a los jóvenes. Se indicaba a los sujetos que para expresar sus respuestas escribiesen el nombre de cada una de esas categorías en el lugar que le atribuyesen en la escala. Por lo demás, se precisaba a los sujetos que podian situar en la misma posición (misma casilla de la escala) todas las categorías que deseasen.

\section{RESULTADOS}

\section{LOS DIFERENTES INDICES DE LOS CUESTIONARIOS}

Antes de pasar a comentar los resultados en función de las diferentes condiciones experimentales, vamos a presentar los diversos indices sobre los que será analizado cada cuestionario.

\subsection{Indices de la influencia}

Como ya se mencionó, este cuestionario se organiza sobre dos dimensiones globales, una directa referida al aborto y la otra indirecta a los anticonceptivos. Los resultados referidos a estas dos dimensiones están constituidos, por una parte, por la media de los 18 items referidos al aborto (cf. anexo, dimensión directa), esta escala del aborto es altamente fiable como lo muestra el alfa de Cronbach de 0,93 que le corresponde. Por otra parte, se ha calculado la media de los 7 ítems referidos a los anticonceptivos (cf. anexo, dimensión indirecta) y que arrojan un alfa de Cronbach de 0,84 , por lo que esta escala de los anticonceptivos es también altamente fiable.

Puesto que la fuente minoritaria defiende una posición favorable al aborto, su influencia directa deberá traducirse por un mayor acuerdo con los items relativos al aborto y su influencia indirecta por mayor acuerdo con los items referidos a los anticonceptivos (asi pues, una puntuación más elevada sobre la escala de 7 puntos).

\subsection{Análisis de la representación de la fuente}

Como se recordará este cuestionario está compuesto de 30 escalas bipolares organizadas en torno a varias dimensiones. Se ha procedido a analizar este cuestionario mediante un análisis factorial en componentes principales con rotación VARIMAX (programa SPSS), cuyos resultados se presentan en el cuadro 1. Este análisis desglosa seis factores que pasamos a presentar.

\subsubsection{Eje 1: evaluación general}

El primer factor, de naturaleza esencialmente evaluativa, satura la mayoría de los items que componen el cuestionario de la imagen de la fuente (concretamente, de los 30 items que componen el cuestionario, están saturados significativamente 21 ). Se encuentran así ítems referidos a la dimensión moral (entre paréntesis figura el coeficiente factorial): inmoral $(-80)$, responsable $(+85)$, egoista $(-70)$, religioso $(+54)$, humanitario $(+50)$. Otra seric de items corresponden a la consistencia de la fuente: incoherente $(-79)$, seguro de sí mismo $(+46)$; a la credibilidad de la fuente: convincente $(+84)$, digno de fe $(+76)$, competente $(+75)$ y portavoz $(+50)$. También un cierto estilo abierto de la fuente se encuentra incluido en este primer factor: democrático $(+61)$, abierto $(+55)$, tolerante $(+41)$, transigente $(+30)$. Se encuentran de igual modo items referidos a la aprobación social: maduro $(+77)$, mayoritario $(+48)$ y marginal $(-43)$. Se encuentran también ciertos items referidos a la consistencia posicional: realista $(+68)$, no objetivo $(-55)$ y crítico $(+34)$.

Así puẹs, en este primer factor no sobresale ninguna dimensión concreta que no esté relacionada con una evaluación general más o menos positiva. 


\section{CUADRO 1}

Coeficientes factoriales del análisis en componentes principales del cuestionario de la imagen de la fuente después de haber realizado una rotación ortogonal varimax $(I=203, J=30)$. Los coeficientes han sido redondeados y multiplicados por cien. Los coeficientes retenidos para el análisis están señalados por un asterisco*.

\begin{tabular}{|c|c|c|c|c|c|c|c|c|}
\hline Item & c & ele $1^{(0)}$ & eje 2 & eje 3 & eje 4 & eje 5 & ele 6 & media \\
\hline $\begin{array}{l}\text { Seguro } \\
\text { Humanitario } \\
\text { Portavoz } \\
\text { Reaccionario } \\
\text { Viejo } \\
\text { Maduro } \\
\text { Incoherente } \\
\text { Mayoritario } \\
\text { Competente } \\
\text { Transigente } \\
\text { Marginal } \\
\text { Digno de fe } \\
\text { Democrático } \\
\text { Tolerante } \\
\text { Feminista } \\
\text { Religioso } \\
\text { Interesado } \\
\text { Realista } \\
\text { Atrevido } \\
\text { Inmoral } \\
\text { Egoista } \\
\text { Crítico } \\
\text { Abierto } \\
\text { No objetivo } \\
\text { Rigido } \\
\text { Responsable } \\
\text { Convincente } \\
\text { Partidista } \\
\text { Independiente } \\
\text { Individualista }\end{array}$ & $\begin{array}{l}38 \\
49 \\
34 \\
22 \\
39 \\
66 \\
71 \\
38 \\
61 \\
49 \\
29 \\
63 \\
64 \\
53 \\
33 \\
59 \\
38 \\
67 \\
35 \\
78 \\
64 \\
30 \\
49 \\
41 \\
60 \\
77 \\
81 \\
46 \\
54 \\
41 \\
\end{array}$ & $\begin{array}{r}46^{*} \\
50^{*} \\
50^{*} \\
-23 \\
-19^{*} \\
77^{*} \\
-79^{*} \\
48^{*} \\
75^{*} \\
30^{*} \\
-43^{*} \\
76^{*} \\
61^{*} \\
41^{*} \\
01^{*} \\
54^{*} \\
-02 \\
68^{*} \\
-19^{*} \\
-85^{*} \\
-70^{*} \\
34^{*} \\
55^{*} \\
-55^{*} \\
-28 \\
85^{*} \\
84^{*} \\
-01^{*} \\
15 \\
04^{*}\end{array}$ & $\begin{array}{c}01 \\
25^{*} \\
15 \\
-09 \\
02 \\
17 \\
-13 \\
36^{\star} \\
17 \\
62^{\star} \\
-30^{\star} \\
18 \\
37^{\star} \\
54^{\star} \\
06 \\
17 \\
-03 \\
20 \\
-01 \\
-14 \\
-34^{\star} \\
03 \\
23 \\
-10 \\
-65^{\star} \\
20 \\
23 \\
-08 \\
01 \\
06\end{array}$ & $\begin{array}{c}30^{*} \\
00 \\
10 \\
-34^{*} \\
-58^{*} \\
08^{*} \\
-21^{*} \\
-03 \\
11 \\
05 \\
-04 \\
12^{*} \\
31^{*} \\
17 \\
56^{*} \\
-38^{*} \\
-01 \\
35^{*} \\
35^{*} \\
16 \\
-06 \\
30^{*} \\
33^{*} \\
-26^{*} \\
04 \\
-05 \\
12 \\
09 \\
17 \\
07^{*}\end{array}$ & $\begin{array}{r}12 \\
14 \\
-19 \\
-09 \\
-12 \\
09 \\
-09 \\
-07 \\
09 \\
01 \\
06 \\
-04 \\
07 \\
05 \\
-09 \\
00 \\
-07 \\
09 \\
12 \\
-07 \\
-12 \\
-14 \\
11 \\
-02 \\
-24 \\
09 \\
12 \\
-66^{*} \\
65 \\
56^{*} \\
\end{array}$ & $\begin{array}{r}09 \\
13 \\
-13 \\
15 \\
06 \\
06 \\
07 \\
-09 \\
-05 \\
-08 \\
03 \\
-03 \\
00 \\
13 \\
08 \\
34 \\
61^{*} \\
08 \\
30 \\
-08 \\
08 \\
-08 \\
-08 \\
09 \\
00 \\
01 \\
-12 \\
08 \\
-23 \\
22 \\
\end{array}$ & $\begin{array}{r}23 \\
38^{*} \\
-04 \\
13 \\
-06 \\
12 \\
09 \\
08 \\
02 \\
06 \\
-10 \\
01 \\
18 \\
09 \\
00 \\
09 \\
03 \\
18 \\
-29{ }^{*} \\
07 \\
-11 \\
-26{ }^{*} \\
-01 \\
15 \\
18 \\
-02 \\
02 \\
-09 \\
11 \\
-18 \\
\end{array}$ & $\begin{array}{r}2,46 \\
3,70 \\
3,35 \\
5,07 \\
6,11 \\
3,61 \\
4,63 \\
4,70 \\
3,53 \\
3,80 \\
4,57 \\
3,63 \\
2,75 \\
3,27 \\
2,60 \\
5,12 \\
3,24 \\
2,65 \\
2,70 \\
4,12 \\
3,86 \\
3,27 \\
2,74 \\
4,76 \\
4,09 \\
3,58 \\
3,54 \\
4,10 \\
3,14 \\
3,85 \\
\end{array}$ \\
\hline $\begin{array}{l}\text { \% de la varianza } \\
\text { común explicada } \\
\% \text { de la varianza } \\
\text { total explicada }\end{array}$ & $\begin{array}{l}100 \\
50,97\end{array}$ & $\begin{array}{l}66 \\
28,19\end{array}$ & 10,6 & 6,06 & 5,5 & 2,86 & 2,07 & \\
\hline
\end{tabular}

(a) eje 1: "evaluación general»; eje 2: “abertura de espiritu»; eje 3: “joven feminista»; eje 4: "la autonomía»; eje 5: «interesado»; eje 6: «moderación social».

\subsubsection{Eje 2: la abertura de espiritu}

Los cuatro primeros items que se encuentran saturados en este segundo factor son: flexible (cf. rígido $[-65]$ ), transigente $(+62)$, tolerante $(+54)$ y democrático (+37). Estos ítems marcan claramente el significado del factor, referido al estilo de negociación (cf. Mugny, 1981). Se complementa con dos ítems de aprobación social: mayoritario $(+36)$ e integrado (cf. marginal $[-30]$ ) y de aprobación moral: generoso (cf. egoísta
[-34]) y humanitario (+25). Visto el carácter de expansividad hacia el otro que está contenido en este factor, nos parece adecuado considerarlo como indicativo del grado de «abertura de espíritu» atribuido a la fuente.

\subsubsection{Eje 3: joven feminista}

Los dos primeros ítems, joven (cf. viejo $[-58])$ y feminista $(+56)$, contenidos en este factor le confieren el sentido. A este «joven feminista» se le asocian ca- 
racterísticas como no religioso (cf. religioso [-38]), progresista (cf. reaccionario $[-34])$, democrático $(+31)$ y la consistencia posicional con los items realista $(+35)$, seguro de sí mismo $(+30)$, objetivo (cf. no objetivo $[-26]$ ) y descendiendo un poco más en los coeficientes se encuentra tambiën coherente (cf. incoherente $[-21]$ ). Se puede apreciar además cierto carácter combativo en los items atrevido $(+35)$ y crítico $(+30)$. Parece quedar, pues, bastante clara la calificación asociada a este «joven feminista» que, podríamos decir, defiende consistentemente una postura progresista $y$ crítica.

\subsubsection{Eje 4: la autonomia}

Los tres ítems contenidos en el factor, autónomo (cf. partidista $[-66]$ ), independiente $(+65)$ e individualista $(+56)$, no dejan lugar a dudas sobre la dimensión de la autonomía a la que se refiere este factor.

\subsubsection{Otros factores (ejes 5 y 6 )}

Los dos últimos factores que nos quedan por ver son menos claros para interpretar. Se tiene así el factor 5 con los items interesado $(+61)$, religioso $(+34)$ y atrevido $(+30)$ que resaltan el interés que moveria al grupo, probablemente en el mismo sentido de una atribución interna como lo refiere Maass y cols. (1982). El factor 6 está compuesto por los items humanitario $(+38)$, no atrevido (cf. atrevido $[-29]$ ) y acrítico (cf. crítico [-26]). Nos referiremos asi a un factor de "moderación social», con toda la prudencia necesaria.

Estas son, pues, las seis dimensiones del cuestionario de la imagen de la fuente sobre las que serán analizadas las distintas condiciones experimentales. Los cuatro primeros factores, los más importantes (explican un 46 por 100 de la varianza total explicada), no se ofrecen a ninguna duda, quedando los dos últimos menos claros. Merece ser señalado que en el primer factor están comprendidos los ítems según el grado de connotación (positiva/negativa) que conlleven. Por el contrario, en los restantes factores los items parecen estar ya más presentes por su denotación específica.

Precisemos finalmente que las puntuaciones factoriales del cuadro 3 están presentadas de tal forma que un signo positivo reenvia a una evaluación general positiva de la fuente (eje 1), a una mayor atribución de abertura de espiritu (eje 2), al reconocimiento de la fuente como joven feminista progresista (eje 3) y a juzgarla como autónoma (eje 4). Para los dos últimos factores, el signo positivo indicará una atribución de interés personal a la fuente (eje 5), así como (y ahora se trata de ser muy prudentes) cierta moderación social (eje 6).

\subsection{Indices del cuestionario de la representación del campo social}

Como se recordará, los sujetos debían colocar en una misma escala de 11 puntos ocho entidades sociales (el sujeto comenzaba situándose a sí mismo y a continuación situaba las siguientes entidades: al grupo minoritario, al gobierno, a la mayoría de la gente, a la Iglesia, a los hombres, a las mujeres y a los jóvenes) según la opinión que ellos creyesen que más caracterizaba a cada una de esas entidades respecto a la legalización del aborto, la gratuidad del aborto, la publicidad del aborto, la utilización de los anticonceptivos y la publicidad de los anticonceptivos. Al igual que para el cuestionario de opiniones, se han construido dos índices según el contenido: uno referido al aborto, compuesto por la media de los tres items referidos al aborto (la legalización, la gratuidad y la publicidad del aborto) y otro referido a los anticonceptivos compuesto por la media de los items referidos a los anticonceptivos (la utilización y la publicidad de los anticonceptivos).

Con afán de simplificación, las diversas entidades han sido también agrupadas a posteriori según estén situadas o no en posiciones próximas. Se tiene así 
que la categoría "gobierno», «la mayoria» y "los hombres» se encuentran siempre situadas entre ellas en posiciones cercanas (se entiende, no hay diferencias significativas entre ellas a nivel estadístico); este conjunto de entidades lo nombraremos categoria mayoría, por contraste con las entidades de "los jóvenes» y «las mujeres", que serán también agrupadas puesto que tampoco difieren entre ellas y lo referiremos como categoría joven-mujer. Estos nombres de categoría mayoría y joven-mujer son totalmente arbitrarios y responden únicamente a la posición que le es atribuida en la escala de 11 puntos, que va desde totalmente desfavorable (caso de la Iglesia) a totalmente a favor. Se encuentra así que sobre la dimensión del aborto las categorías se ordenan de esta forma (de más opuesto a más a favor): la Iglesia ( $m=1,85$ ), la categoría mayoría ( $\mathrm{m}=7,22)$, la categoría jovenmujer $(\mathrm{m}=8,56)$ y el grupo minoritario $(\mathrm{m}=8,87)$. En este contexto el texto propuesto a los sujetos aparece claramente como minoritario y, sobre todo, si se tiene en cuenta que el sujeto mismo se sitúa por debajo de la categoría mayoria. Conviene precisar, pues, que en el análisis de los resultados habrá que tener en cuenta que en realidad las categorias no son puramente descriptivas (más o menos favorables) sino que reenvían de hecho a diferentes subgrupos cuya significación para la identificación social será muy diferente, sobre todo porque todos los sujetos de este experimento pertene- cen al mismo sexo (femenino, concretamente).

\subsection{Resultados experimentales propiamente dichos}

Los sujetos respondieron en primer lugar al pretest. La media global de los ítems que componen este cuestionario es de 4,29. Como era de esperar, las diversas condiciones no difieren entre sí, lo que nos coloca en una situación ideal, pues, para estar seguros que toda diferencia entre condiciones se deberá a la manipulación experimental introducida.

El análisis de los resultados siguientes lo vamos a presentar según las diversas cuestiones que se trataban de examinar en este experimento. Así se realizarán las comparaciones entre condiciones que sean pertinentes para cada una de las cuestiones examinadas. Por último, precisamos que los niveles estadísticos de significación son en general bilaterales, en caso contrario se precisarán las comparaciones unilaterales.

\subsubsection{El efecto "directo» del texto sobre las opiniones}

Como se recordará, en la condición fuente-opinión los sujetos contestaron al cuestionario de opiniones, referido al aborto y a los anticonceptivos, inmediatamente después de haber leído el texto favorable a la legalización y a la gratui-

\section{CUADRO 2}
Puntuaciones medias para las dimensiones del aborto y los anticonceptivos sobre la escala de 7 puntos $(1=$ totalmente en desacuerdo, $7=$ totalmente de acuerdo) del cuestionario de opiniones. Entre paréntesis ( $n$ ) figura el número de sujetos por condición y la desviación típica.

\begin{tabular}{lllllll}
\hline & & \multicolumn{4}{c}{ dimensión } \\
\cline { 3 - 7 } & $(\mathbf{n})$ & \multicolumn{3}{c}{ aborto } & \multicolumn{2}{c}{ anticonceptivos } \\
\hline control & 53 & 3,78 & & $(1,31)$ & 5,06 & $(1,33)$ \\
fuente-opinión & 29 & 3,63 & $(1,40)$ & 5,05 & $(1,31)$ \\
fuente-imagen & 39 & 4,00 & $(1,26)$ & 5,54 & $(0,98)$ \\
fuente intragrupo & 42 & 3,56 & $(1,41)$ & 4,87 & $(1,40)$ \\
fuente extragrupo & 40 & 4,34 & $(1,43)$ & 5,35 & $(1,23)$ \\
\hline
\end{tabular}




\section{CUADRO 3}

Medias de las puntuaciones factoriales de cada condición experimental en cada uno de los 6 factores del cuestionario de la imagen de la fuente (cf. cuadro 1). Una signo positivo indica que más se aplica la dimensión mencionada.

\begin{tabular}{lcccccc}
\hline & eje 1(a) & eje 2 & eje 3 & eje 4 & eje 5 & eje 6 \\
\hline control & -.10 & +.08 & -.35 & +.03 & -.07 & -.08 \\
fuente-opinión & +.04 & -.07 & -.19 & +.10 & -.08 & -.18 \\
fuente-imagen & +.05 & +.12 & +.27 & -.03 & -.05 & -.02 \\
fuente intragrupo & -.15 & -.30 & +.27 & -.02 & +.20 & +.23 \\
fuente extragrupo & +.21 & +.14 & +.05 & -.05 & 00 & +.01 \\
\hline
\end{tabular}

(a) eje 1: "evaluación general»; eje 2: “abertura de espiritu»; eje 3: «joven feminista»; eje 4: "la autonomia»; eje 5: “interesado"; eje 6: “moderación social».

\section{CUADRO 4}

Puntuaciones medias de la escala de 11 puntos $(1=$ totalmente en contra, 11 = totalmente a favor) de las categorias del cuestionario de la representación del campo social.

\section{A) DIMENSION DEL ABORTO}

\begin{tabular}{lccccc}
\hline & \multicolumn{5}{c}{ categorias } \\
\cline { 2 - 6 } & sujeto & texto & joven-mujer & mayoria & iglesia \\
\hline control & 5,04 & 6,17 & 8,47 & 7,14 & 1,94 \\
fuente-opinión & 5,62 & 8,07 & 8,71 & 7,45 & 1,60 \\
fuente-imagen & 6,81 & 9,77 & 8,82 & 7,50 & 2,02 \\
fuente intragrupo & 5,52 & 9,90 & $\mathbf{8 , 2 5}$ & 6,91 & 1,85 \\
fuente extragrupo & 6,98 & 10,42 & $\mathbf{8 , 5 6}$ & 7,11 & 1,83 \\
\hline
\end{tabular}

\section{A) DIMENSION DE LOS ANTICONCEPTIVOS}

\begin{tabular}{lccccc}
\hline & \multicolumn{5}{c}{ categorias } \\
\cline { 2 - 6 } & sujeto & texto & joven-mujer & mayoria & iglesia \\
\hline control & $\mathbf{8 , 0 1}$ & 6,17 & 9,27 & 8,11 & 2,35 \\
fuente-opinion & 7,76 & 6,84 & 8,96 & 8,20 & 1,60 \\
fuente-imagen & 9,24 & $\mathbf{8 , 5 3}$ & 9,90 & 8,32 & 2,23 \\
fuente intragrupo & 7,52 & $\mathbf{9 , 0 2}$ & 9,35 & 7,91 & 2,51 \\
fuente extragrupo & $\mathbf{8 , 4 5}$ & $\mathbf{9 , 0 7}$ & 9,43 & $\mathbf{8 , 3 5}$ & $\mathbf{1 , 9 1}$ \\
\hline
\end{tabular}

dad del aborto atribuido a un grupo minoritario de jóvenes. Para conocer el impacto directo del texto sobre las opiniones de los sujetos, esta condición fuenteopinión se compara a la condición control en la que los sujetos comienzan contestando al cuestionario de opiniones sin haber leído el texto. Los resultados sobre el cuestionario de opiniones (véase cuadro 2) muestran que los sujetos de la condición fuente-opinión que leyeron el texto a favor del aborto, expresan una actitud frente al aborto ( $\mathrm{m}=3,63$ ) semejante a los de la condición control (m
$=3,78)$. De igual modo, sobre la dimensión de los anticonceptivos, los sujetos de la condición fuente-opinión $(\mathrm{m}=$ $5,05)$ no difieren de los de la condición control ( $\mathrm{m}=5,06 ; \mathrm{t}[198]=0,053$ n.s.). Así pues, el impacto del texto minoritario en esta condición fuente-opinión es nulo respecto a la condición control.

¿Qué ocurre sobre la imagen que emiten los sujetos de la condición fuenteopinión del grupo minoritario de jóvenes, autor del texto que leyeron, comparada a la emitida por los de la condición 


\section{Estudios}

control, que responden al cuestionario imaginándose un grupo minoritario de jóvenes que estuviese a favor de la legalización y la gratuidad del aborto, pero sin haber leído ningún texto? Las puntuaciones factoriales correspondientes a estas dos condiciones (véase cuadro 3) no difieren estadísticamente hablando, en ninguna de las seis dimensiones del análisis factorial.

¿Cómo interpretar la ausencia de diferencias entre estas dos condiciones, tanto a nivel de las opiniones como de la imagen?

La primera respuesta vendría a la mente sin esfuerzo: el texto minoritario no es portador de ninguna alternativa, ya sea porque estar a favor de la legalización y la gratuidad del aborto no es una postura suficientemente minoritaria, ya sea porque la consistencia (tal como se refirió anteriormente) no está bien operacionalizada en la "retórica» del texto. En este caso, si el grupo minoritario no constituye una entidad social bien diferenciada y no es portador de una alternativa minoritaria, resulta lógico que no haya obtenido influencia.

La medida de la representación del campo social resulta ser totalmente apropiada para examinar este punto. ¿Sitúan los sujetos al grupo minoritario «imaginario" (condición control) y al grupo minoritario autor del texto en la misma posición en el conjunto del campo social? El interés de esta cuestión es simple: por una parte, si el grupo minoritario real no es situado más a favor del aborto que el grupo minoritario «imaginario", entonces hay que concluir que la «retórica» del texto no ha sido eficiente. Por otra parte, para poder decir que el grupo minoritario real es concebido como una verdadera entidad minoritaria portadora de una alternativa innovadora, deberiamos encontrar que el grupo minoritario real es situado más a favor del aborto que cualquier otra categoría social. Examinemos a la luz de los datos del cuadro 4 esta indagación.
Si comparamos en primer lugar las dos condiciones que nos han hecho desembocar en este examen de los resultados, se tiene que los sujetos de la condición control sitúan al grupo minoritario (imaginario) menos a favor de la legalización y la gratuidad del aborto $(\mathrm{m}=$ 6,17 ) de lo que es situado el grupo minoritario real por los sujetos de la condición fuente-opinión $(\mathrm{m}=8,07, \mathrm{t}[198]$ $=3,273 ; \mathrm{p}<0,001)$. Así pues, lo menos que se puede decir aqui es que la posición del grupo minoritario, favorable a la legalización y gratuidad del aborto, ha sido bien percibida por los sujetos que leyeron el texto y por lo tanto se confirma que la consistencia está bien operacionalizada. El segundo punto era saber si el grupo minoritario constituía una entidad social bien diferenciada y alternativa (la más favorable al aborto, pues). Comparémoslo con las restantes entidades. Se encuentra así que en la condición control el grupo minoritario imaginario ( $m=6,17)$ es situado menos a favor del aborto que la categoría mayoría $(\mathrm{m}=7,14, \mathrm{t}[52]=1,76 ; \mathrm{p}$ $<0,09)$ y que la categoría joven-mujer $(\mathrm{m}=8,47, \mathrm{t}[52]=4,21 ; \mathrm{p}<0,001)$, por lo que en esta condición no es visto como la entidad más favorable al aborto. En la condición fuente-opinión el grupo minoritario es situado con esas dos categorias: la categoría joven-mujer ( $\mathrm{m}=$ 8,71 ) no difiere de la posición atribuida al grupo minoritario $(\mathrm{m}=8,07 ; \mathrm{t}[28]=$ 0,84 ; n.s.) y la categoría mayoría ( $\mathrm{m}=$ $7,45)$ tampoco difiere del grupo minoritario en esta condición $\mathrm{t}(28)=0,81$; n.s.). No obstante, y esto es de suma importancia, el grupo minoritario no constituye aún la categoría social más favorable al aborto.

Si se observan las otras tres condiciones restantes (fuente-imagen, intragrupo y extragrupo) se tiene que el grupo minoritario es situado más a favor del aborto que ninguna otra categoría. En concreto las puntuaciones correspondientes al grupo minoritario son: 9,77 para la condición fuente-imagen, que difiere de la categoría joven-mujer $(\mathrm{m}=$ 
$8,82, \mathrm{t}[38]=2,27 ; \mathrm{p}<0,03)$, que es la categoría que más se le aproxima. En la condición intragrupo la media del grupo minoritario es de 9,90 , que también difiere de la categoría joven-mujer ( $\mathrm{m}=$ $8,25, \mathrm{t}[41]=3,26 ; \mathrm{p}<0,002)$ y de igual modo en la condición extragrupo el grupo minoritario $(\mathrm{m}=10,42)$ es la categoría más favorable, diferenciándose significativamente de la categoria jovenmujer $(\mathrm{m}=8,56, \mathrm{t}[39]=5,90 ; \mathrm{p}<$ 0,001 ), que le sigue a continuación.

Estos resultados nos demuestran dos cosas: por una parte que la posición defendida en el texto minoritario es claramente percibida como favorable al aborto (en este caso la ausencia de diferencia encontrada entre la condición control y la condición fuente-opinión no puede ser explicada diciendo que la reivindicación del grupo minoritario no es percibida como tal). Pero al mismo tiempo, estos resultados muestran que, a pesar de todo, en la condición fuente-opinión, el grupo minoritario no es percibido como la entidad social más favorable al aborto, contrariamente a las condiciones fuenteimagen, intragrupo y extragrupo. Esto nos llevan a reflexionar sobre dos puntos.

Por una parte, hay que preguntarse por qué la diferencia entre la condición fuente-opinión y la condición control en cuanto al reconocimiento de la posición de la alternativa defendida por el grupo minoritario no se traduce en un efecto diferencial de influencia sobre las opiniones de los sujetos, tal como se traduce, como se verá, la diferencia entre dicha condición y la condición fuente-imagen. Un elemento de explicación verosímil puede encontrarse en la forma en la que está construido el cuestionario de opiniones. En efecto, todos los ítems están redactados de forma afirmativa a favor del aborto y de los anticonceptivos por lo que no se puede excluir un efecto persuasor en la mera presentación («mere exposure», Vanbeselaere, 1983) de los items. Para verificar la existencia de este posible efecto hemos comparado los cinco items del pretest con esos mismos cinco items del cuestionario de opiniones en la condición control (ésta es la única condición en la que los sujetos no leyeron el texto minoritario). Nuestro razonamiento es el siguiente: si en los cinco ítems del cuestionario de opiniones los sujetos se muestran más de acuerdo con la dimensión del aborto que en los cinco del pretest, entonces existiria el efecto de mera presentación. Este resulta ser el caso. Los sujetos en esta condición control tienden ligeramente (en hipótesis unilateral este efecto sería casi significativo) a mostrarse menos de acuerdo con los cinco items del pretest ( $\mathrm{m}=4,17$ ) que con esos mismos cinco items contenidos en el cuestionario de opiniones $\mathrm{m}$ $=4,26 ; \mathrm{t}(52)=1,60: \mathrm{p}<0,12)$. En este caso, el grado de reconocimiento de la posición favorable al aborto manifestado por los sujetos de la condición fuente-opinión es insuficiente para adicionar su efecto al provocado por la mera presentación de los ítems.

El segundo aspecto, posiblemente explicativo de la no diferencia entre estas dos primeras condiciones experimentales, se refiere al importante papel que parece tener la elaboración de una representación del grupo minoritario antes de que el sujeto pase a expresar sus propias opiniones. Como se recordará hay una diferencia global en el orden de respuesta al cuestionario de opiniones entre la condición fuente-opinión (los sujetos responden al cuestionario de opiniones y representación del campo social antes de responder al cuestionario de la representación de la fuente) y las tres restantes en las que los sujetos responden al cuestionario de la representación de la fuente antes de los otros dos cuestionarios. En el siguiente apartado analizaremos este aspecto.

Para completar los puntos tratados en este apartado, conviene comentar lo que ocurre con la representación del campo social sobre la dimensión de los anticonceptivos en la que se observan dos resultados de sumo interés: en primer lugar se observa que cuanto más a favor del aborto fue situado el grupo minoritario 
en una condición determinada, más es ahora situado en esa condición a favor de los anticonceptivos, como nos lo muestra la correlación $(\mathrm{r}=0,73 ; \mathrm{p}<$ $0,001)$ entre esas dos dimensiones sobre dicha escala. Se encuentran, pues, las mismas diferencias entre las condiciones que las vistas en la dimensión del aborto. A saber: que en la condición control el grupo minoritario tiende a ser situado menos a favor de los anticonceptivos (m $=6,17$, esta media coincide exactamente con la de la dimensión del aborto) que en la condición fuente-opinión ( $\mathrm{m}=$ 6,84 ), si bien esa diferencia no llega a alcanzar un nivel aceptable de significación estadística. Y en la condición fuente-opinión el grupo minoritario es situado menos a favor de los anticonceptivos que en las otras condiciones restantes las cuales no difieren entre si $(\mathrm{m}=8,53$ para la condición fuente-imagen que difiere de la condición fuente-opinión $\mathrm{t}(198)=2,362 \mathrm{p}<0,02 ; \mathrm{m}=9,02$ para la condición intragrupo, que difiere igualmente de la condición fuente-opinión $\mathrm{t}(198)=3,110 \mathrm{p}<0,006 \mathrm{y} \mathrm{m}=$ 9,07 para la condición extragrupo, que también difiere de la condición fuenteopinión $t(198)=3,151 p<0,005)$. El segundo resultado que sobresale en esta dimensión de los anticonceptivos es que el grupo minoratario deja de ser la categoría más favorable (lo contrario, pues, de lo visto en la escala del aborto). En esta dimensión de los anticonceptivos el grupo minoritario sobresale menos como entidad alternativa en el campo social. Se constata así un aspecto que no habrá que olvidar a partir de ahora para seguir la exposición de los resultados: el sujeto infiere perfectamente que cuanto más a favor del aborto estuvo el grupo minoritario, más lo estará a favor de los anticonceptivos, luego la dimensión de los anticonceptivos sigue siendo una dimensión sobre la que se encuentra definida la posición del grupo minoritario y por lo tanto resulta correcto decir que cuanto más a favor de los anticonceptivos esté el sujeto más ha sido influenciado por el grupo minoritario. Pero al mismo tiempo, el grupo minoritario, en tanto que entidad social, en esta dimensión sobresale menos en el campo social por encima del resto de las categorías sociales.

Así pues, se fundamenta nuestra conjetura según la cual la escala de los anticonceptivos constituye una dimensión indirecta, $y$ es indirecta justamente porque sobre esta dimensión la identificación con el grupo minoritario será menos conflictiva. De cualquier modo, quedará por estudiar por qué los sujetos infieren (a partir del texto sobre el aborto) perfectamente la posición del grupo minoritario frente a los anticonceptivos y que al mismo tiempo lo intengran en tanto que entidad social en el conjunto del campo social, como si se tratase de un efecto de "desdramatización», a fin de que perdiera sus características "comprometedoras».

\subsubsection{La representación de la fuente: un proceso mediacional de la influencia minoritaria}

En la condición fuente-imagen los sujetos, inmediatamente después de haber leído el texto del grupo minoritario de jóvenes, pasan a contestar al cuestionario de la imagen de la fuente. La comparación de esta condición con la condición fuente-opinión, nos permite abordar de modo estricto el papel hipotetizado que puede representar la imagen como mediadora en los procesos de influencia minoritaria.

Consideremos, en primer lugar, las opiniones frente al aborto y los anticonceptivos, es decir, los efectos de influencia. Sobre la dimensión del aborto, los sujetos que describieron la fuente antes de expresar sus opiniones, tienden ligeramente a estar más a favor del aborto ( $m$ $=4,00$ ) que los que la describen después $(\mathrm{m}=3,63)$, si bien esta diferencia no alcanza el nivel estadístico de significación aceptable. Esta tendencia se acentúa sobre la dimensión de los anticonceptivos, donde los sujetos que comienzan describiendo la fuente mostrarán después opiniones más favorables a los anticonceptivos ( $m=5,54$ ) que los suje- 


\section{Estudios}

tos que responden de entrada al cuestionario de opiniones $(\mathrm{m}=5,05 ; \mathrm{t}(198)$ $=1,578 ; \mathrm{p}<0,06$, hipótesis unilateral). Globalmente, los sujetos expresan opiniones más cercanas a la fuente cuando, una vez leído el texto minoritario a favor del aborto, pasan a expresar la imagen de la fuente antes de expresar sus propias opiniones. Se ha confirmado nuestra hipótesis según la cual habría más influencia cuando los sujetos elaboran la imagen entre la lectura del texto minoritario y la expresión de las opiniones.

¿Por qué? Examinemos la imagen emitida por los sujetos cuando lo hacen antes o después de expresar sus propias opiniones. Sólo el factor joven feministo diferencia estas dos condiciones (véase el cuadro 3). Los sujetos de la condición fuente-imagen perciben el grupo minoritario más «joven feminista» ( $m=0,27$ ) que los sujetos de la condición fuenteopinión $(\mathrm{m}=-0,19 ; \mathrm{t}(198)=2,240 ; \mathrm{p}<$ $0,03)$. Ahora bien, como se dijo, a esta dimensión joven-feminista le están asociadas tanto la consistencia como el carácter crítico y progresista. No creemos equivocarnos al decir que el grupo minoritario ha sido percibido con una entidad progresista innovadora. Para saberlo de otra forma, examinemos dónde es situado el grupo minoritario en la escala de la representación del campo social. En efecto, ya hemos dicho que los sujetos de la condición fuente-imagen en esta escala de la representación del campo social (véase el cuadro 4) sitúan al grupo minoritario más a favor del aborto $(\mathrm{m}=9,77)$ que los de la condición fuente-opinión ( $\mathrm{m}=8,07$ ) y también más a favor de los anticonceptivos $(\mathrm{m}=$ 8,53 para la condición fuente-imagen y $\mathrm{m}=6,84$ para la condición fuente-opinión. Su reivindicación es percibida más a favor del aborto. De igual modo, dentro del conjunto del campo social, la fuente minoritaria es situada más a favor del aborto que cualquier otra categoria (cf. cuadro 4), lo que no es el caso en la condición fuente-opinión. Luego en la condición fuente-imagen el grupo minoritario es la categoria social que repre- senta la posición más alternativa frente al aborto. Se tiene, pues, que en la condición en la que hay más influencia la fuente minoritaria ha sido percibida también como más alternativa. Se vuelve a encontrar de nuevo una verificación más, por una parte, que la minoría para obtener influencia tiene que ser percibida como alternativa (contranorma) y, por otra, del importante papel que juega el conflicto introducido por la minoria al presentarse como alternativa en el campo social (cf. Moscovici, 1979 y Mugny, 1981), puesto que la influencia aumenta cuando aumenta la distancia - el conflicto, pues- entre la fuente y el resto de las categorias, lo que tiende al mismo tiempo a desplazar la influencia sobre la dimensión indirecta, dimensión en la que el conflicto de identificación se presenta en menor grado, lo que es coherente con la teoria de la influencia minoritaria como «conversión» (cf. Moscovici, 1980).

Para terminar este apartado examinemos la dimensión de los anticonceptivos sobre la representación del campo social. Se tiene que el sujeto en esta escala se sitúa a sí mismo más a favor de los anticonceptivos en la condición fuente-imagen $(\mathrm{m}=9,24)$ que en la condición fuente-opinión $(\mathrm{m}=7,76 ; \mathrm{t}(198)=$ $2,140 ; p<0,04)$, resultado que corrobora el efecto de influencia indirecta sobre esta dimensión en el cuestionario de opiniones. Pero, cómo sitúa el sujeto el resto de las categorias sociales respecto a sí mismo?

Tanto en la condición fuente-imagen como en la condición fuente-opinión el sujeto se sitúa a si mismo más a favor de los anticonceptivos de lo que sitúa al grupo minoritario y se autositúa menos a favor de lo que sitúa a la categoría joven-mujer $(\mathrm{t}(38)=2,24 ; \mathrm{p}<0,04$ para la condición fuente-imagen y $\mathrm{t}(28)=$ 2,$19 ; \mathrm{p}<0,04$ para la condición fuenteopinión). Ahora bien, en la condición fuente-imagen el sujeto mismo está más a favor de los anticonceptivos de lo que sitúa a la categoria mayoría $(\mathrm{t}(38)=$ 3,36; $\mathrm{p}<0,002)$ lo que no es el caso en 
la condición fuente-opinión $(\mathrm{t}(28)=$ 0,85 ), donde el resultado incluso se invierte. Se tiene, pues, que en la condición fuente-imagen el grupo minorario, percibiendo como más joven feminista que en la condición fuente-opinión (visto en el cuestionario de la representación de la fuente y corroborado en el cuestionario de la representación del campo social), ha provocado un efecto de diferenciación con la categoría mayoría. ¿ ¿No es éste un índice de redefinición de la identidad psicosocial del sujeto en el conjunto del campo social provocado por la intervención de la minoría que ha sido percibida como una categoría más «joven feminista» (opuesta en cierto modo a la categoria mayoria), tal y como se vio en el análisis del cuestionario de la imagen de la fuente y la representación del campo social?

Este análisis sobre el papel de la imagen en los fenómenos de influencia, nos ha hecho desembocar en la división del campo social puesto en juego en los procesos de influencia social. En el punto siguiente se trata de observar los efectos de la categorización social introducida esta vez explícitamente a título de variable independiente.

\subsubsection{Efecto de la comunidad y diferencia de pertenencias categoriales}

En la condición fuente intragrupo se precisaba que el autor del texto era un grupo minoritario de jóvenes mujeres, con la misma pertenencia sexual que el sujeto. En la condición fuente extragrupo el texto fue atribuido a un grupo minoritario de jóvenes bombres, así pues, con distintas pertenencia sexual que los sujetos. Hay un efecto diferencial entre estas dos condiciones en lo que a la influencia se refiere.

Sobre la dimensión directa del aborto se encuentra (veáse cuadro 2) que en la condición fuente extragrupo los sujetos muestran mayor acuerdo con el aborto $(\mathrm{m}=4,34)$ que en la condición fuente intragrupo $(\mathrm{m}=3,56 ; \mathrm{t}[198]=2,605 ; \mathrm{P}$
$<0,01)$. Aunque algo menos elevada, sobre la dimensión de los anticonceptivos se encuentra también que en la condición fuente extragrupo los sujetos muestran mayor acuerdo con los anticonceptivos $(m=5,35)$ que los sujetos de la condición fuente intragrupo ( $\mathrm{m}=$ 4,$87 ; \mathrm{t}[198]=1,728 ; \mathrm{p}<0,09$ ). La condición fuente extragrupo obtiene, pues, más influencia que la condición fuente intragrupo.

Sobre el cuestionario de la representación de la fuente minoritaria, se encuentran dos factores que diferencia estas dos condiciones: sobre el factor evaluatorio se tiene que en la condición fuente extragrupo se tiende a evaluar más positivamente la fuente minoritaria $(\mathrm{m}=+$ $0,21)$ que en la condición fuente intragrupo $(\mathrm{m}=-0,15 ; \mathrm{t}[198]=1,68 ; \mathrm{p}<$ $0,10)$. Pero sobre todo se encuentra que en la dimensión de abertura de espíritu (eje 2) los sujetos de la condición fuente extragrupo perciben la fuente minoritaria más abierta de espíritu $(m=+0,14)$ que la condición fuente intragrupo (m $=-0,30 ; \mathrm{t}[198]=2,408 ; \mathrm{p}<0,02)$. Así pues, los sujetos de la condición fuente intragrupo parecen elaborar una imagen bien precisa de la fuente minoritaria que la evalúan poco positivamente y más dogmática, por oposición abierta de espíritu. Incluso se tendería a verla como más joven feminista (eje $3: \mathrm{m}=$ $+0,27$ versus $+0,05$ en la condición fuente extragrupo), pero un feminismo (podríamos decir) que, además de no ser valorado positivamente y tachado de cierto dogmatismo, es interesado (eje 5: $\mathrm{m}=+0,20$ versus 0,00 para la condición fuente extragrupo), si bien, lamentablemente, estas puntaciones sobre estos dos factores no alcanzan un nivel de significación estadístico aceptable. Los sujetos de la condición fuente intragrupo parecen crear así una división intracategorial («nosotras y ...ellas, las feministas») que explicaría la falta de influencia en esta condición. Lo que para la fuente extragrupo es "abertura de espíritu» pasa a significar «dogmatismo» para la fuente intragrupo. 
¿Cómo se traducen estos aspectos en la representación del campo social? Examinemos en primer lugar la dimensión directa del aborto. Se tiene así que las posiciones (veáse cuadro 4) atribuidas por los sujetos de la condición fuente intragrupo a cada una de las entidades que el sujeto debía incluir en esta escala no difieren de las atribuidas por la condición fuente extragrupo. Sobre esta dimensión, pues la estructuración del campo social es independiente de la comunidad de pertenencias categoriales entre el sujeto y la fuente minoritaria. Pero no ocurre lo mismo con la posición que toma el sujeto mismo dentro de esa estructura del campo social. Se constatan así dos efectos diferenciales. En primer lugar, en la condición fuente intragrupo los sujetos se sitúan menos a favor del aborto $(\mathrm{m}=5,52)$ que los sujetos de la condición fuente extragrupo $(\mathrm{m}=6,98$; $\mathrm{t}[198]=2,135 ; \mathrm{p}<0,04)$, este efecto corrobora, pues, lo visto sobre el cuestionario de opiniones. El segundo efecto se refiere a la distancia que establece el sujeto entre su propia posición y la categoria mayoría. Asi, en la condición fuente intragrupo el sujeto $(m=5,52)$ se distancia significativamente de la categoria mayoria $(\mathrm{m}=6,91 ; \mathrm{t}[41]=2,97$; $\mathrm{p}<0,005)$ lo que no es el caso en la condición fuente extragrupo (la media del sujeto mismo es de 6,91 y 7,11 para la categoria mayoría, $\mathrm{t}[39]=0,29)$. Se podría decir así que, en cierto modo, el sujeto influenciado se apoya en otra categoría para serlo $y$, por el contrario, que el sujeto que no es influenciado continúa "aislándose» dentro del campo social. Ahora bien, ¿cómo explicar esa identificación (o al menos la no diferenciación) de la condición fuente extragrupo con la categoria mayoria? Debemos recordar que en esta categoria mayoría está incluido el grupo de hombres (misma pertenencia sexual que la fuente minoritaria) por lo que parece claro que, aunque el sujeto guarda la distancia con el grupo minoritario (estamos en la dimensión directa), no obstante, no se diferencia de la categoría mayoría que incluye cierta comunidad con la fuente minoritaria. ¡ $\mathrm{Pa}$ - rece tratarse, pues, de una especie de identificación indirecta con la minoría sobre una dimensión directa!

Sobre la dimensión de los anticonceptivos los únicos efectos diferenciales entre la condición fuente intragrupo $y$ fuente extragrupo que merecen ser retenidos se encuentran de nuevo en las mismas entidades que en la dimensión directa del aborto (la posición del sujeto mismo y la categoria mayoria). De igual modo se encuentra que la posición que se autoatribuye el sujeto tiende a corrobar los efectos vistos sobre el cuestionario de opiniones para esta dimensión indirecta de los anticonceptivos. Así, en la condición fuente intragrupo los sujetos $(\mathrm{m}=7,52)$ tienden a situarse menos a favor de los anticonceptivos que en la condición fuente extragrupo $(\mathrm{m}=8,45$; $\mathrm{t}[198]=1,481 ; \mathrm{p}<0,14)$. El segundo efecto surge de la posición que se atribuye el sujeto y la que atribuye a la categoría mayoría. Se tiene así que los sujetos de la condición fuente intragrupo tienden a situar la categoría mayoría menos a favor de los anticonceptivos ( $\mathrm{m}=$ $7,91)$ que los de la condición fuente extragrupo $(\mathrm{m}=8,35 ; \mathrm{t}[198]=1,278 ; \mathrm{p}$ $<0,11)$. Por otra parte, en la condición fuente intragrupo el sujeto mismo $(\mathrm{m}=$ 7,52 ) sigue distanciándose significativamente del grupo minoritario $(\mathrm{m}=9,02$; $\mathrm{t}[41]=2,27 ; \mathrm{p}<0,03$ ), lo que no es el caso en la condición fuente extragrupo en la que el sujeto $(\mathrm{m}=8,45)$ se sitúa entre el grupo minoritario $(\mathrm{m}=9,07)$ y la categoría mayoria $(8,35)$, dos entidades que sigue separando y en medio de las cuales se sitúa el mismo. Para esta condición fuente extragrupo la categoría mayoría parece constituir, pues, una protección a través de la cual el sujeto puede aceptar la posición de la minoría de forma más indirecta en la dimensión del aborto y más manifiesta (no diferenciándose ya del grupo minoritario) sobre la dimensión de los anticonceptivos.

\section{DISCUSION}

Este estudio exploratorio ha cumplido sus objetivos. Respecto al paradigma ex- 
perimental que deseábamos examinar, se pueden extraer varias conclusiones. En primer lugar, las reivindicaciones avanzadas en el texto constituyen evidentemente una posición minoritaria frente al aborto, tal como se vio en la escala de la representación del campo social donde en general la fuente a la que se atribuyó dicho texto fue situada como la entidad social más extrema favorable al aborto.

En segundo lugar, merece destacarse la correlación significativa entre el cuestionario de opiniones y el cuestionario de la representación del campo social: los efectos diferenciales entre condiciones en las opiniones del propio sujeto encontrados en un cuestionario se encuentran de igual forma corroborados en el otro. Así, pues, no sólo las dos escalas son sensibles al mismo tipo de efecto (prueba de la validez de las escalas) sino que además nos aseguran de los efectos encontrados (no muy altos a nivel estadístico, hay que reconocerlo) por dicha reiteración.

Otro aspecto que merece reseñarse es lo referido a la media de la influencia directa e indirecta. A priori se introdujo la dimensión referida al aborto, la única sobre la que se definia manifiestamente la fuente minoritaria (dimensión directa), y la otra referida a los anticonceptivos sobre la que la fuente minoritaria no explicitaba ninguna referencia en el texto que se le atribuyó (dimensión indirecta). $\mathrm{Y}$, como se mencionó, nos fundábamos en dos criterios para establecer esta diferencia: por no estar contenida en el texto y por tratarse de un tema diferente al del aborto, pero con la suficiente probabilidad para que fuese inferida la relación. Esta dinámica la han entendido perfectamente nuestros sujetos. Se ha visto así, a través de la escala de la representación del campo social (dirigida también a captar este proceso), que cuanto más a favor del aborto fue percibido el grupo minoritario, más los sujetos infieren que lo está también a favor de los anticonceptivos. Este paralelismo entre las dos dimensiones nos asegura de que la dimensión de los anticonceptivos es también, indirectamente, por inferencia, pertinente para reconocer la fuente minoritaria. Pero al mismo tiempo, si la posición de la minoría sobre los anticonceptivos está reconocida, sin embargo, los sujetos integran (paradójicamente, en cierto modo) al grupo minoritario en el conjunto del campo social. Sería una forma de desdramatizar el carácter innovador, minoritario, "desviado", contenido en el grupo minoritario. El resultado de este proceso es que sobre la dimensión indirecta el grupo minoritario pierde así su carácter connotador y denotador de baluarte de una identificación social problemática. De este modo todo acercamiento al grupo minoritario se convierte en menos conflictual en dicha dimensión indirecta, puesto que implica la identificación social con la posición minoritaria en un grado inferior.

Vistas estas apreciaciones globales sobre el paradigma experimental, veamos ahora el significado de algunos efectos experimentales que más han resaltado en este experimento. En primer lugar, se verificó nuestra hipótesis según la cual la representación de la fuente minoritaria constituye un proceso mediaçional en los procesos de influencia minoritaria. Se ha visto que cuando en cierto modo el sujeto confronta sus opiniones directamente con la posición de la fuente minoritaria, ésta no produce ningún tipo de influencia, ni sobre la dimensión directa ni la indirecta, como si cada entidad debiera permanecer sobre las posiciones que ha afirmado. Por el contrario, cuando entre la lectura de la posición minoritaria y la expresión por parte del sujeto de sus propias opiniones ha habido una actividad cognitiva sobre la representación de la fuente minoritaria (aspecto a la vez inductivo $e$ inferencial), aparece una influencia. Ahora bien, ésta se encuentra sobre todo en la dimensión indirecta, resultados confirmados respectivamente al examinar la posición del propio sujeto en la escala de la representación del campo social. En este nuevo paradigma se encuentran, pues, efectos similares a los ya existentes en la literatura sobre la influencia de las minorías, donde en diver- 
sas ocasiones ha sido demostrado que el impacto de las minorías se produce sobre todo sobre la dimensión indirecta. Estos resultados adjuntan, pues, una prueba suplementaria al reciente capitulo de la conversión (Moscovici, 1980).

Se vio que la actividad cognitiva sobre la elaboración de la representación de la fuente inmediatamente después de la lectura del texto minoritario se traducía en la atribución la dimensión «jovenfeminista" a la minoría, lo que no era el caso cuando la representación del grupo minoritario se efectuaba después de expresar las opiniones. Una explicación de esta diferencia, probablemente certera, es que en la condición fuente-imagen el sujeto atribuye más significaciones e interpretaciones a la posición de la minoria; en cierto sentido se puede decir que el sujeto va más allá de la posición defendida por la minoria. Elaboraria así una representación con cierta independencia de sus propias opiniones por lo que reconocería el carácter joven-feminista de la fuente que, aunque pueda resultar conflictual en un momento dado (la influencia tiende a aparecer sobre todo a nivel indirecto), sin embargo ha provocado su impacto. Por el contrario, en la condición fuente-opinión el sujeto elabora la representación de la fuente después de haber dejado expresadas sus propias opiniones y no «haberse dejado» influenciar, con lo que la imagen de la fuente estaría entre dos fuegos. Por una parte, si juzga a la fuente positivamente se estaría juzgando a sí mismo negativamente (el sujeto está más en contra del aborto que la fuente minoritaria). Se puede suponer, pues, que el sujeto justifica, en cierto modo, sus propias opiniones anteriormente expresadas y de ahi que no reconozca ciertas características de la fuente que, a pesar de todo, podrían considerarse independientes de la propia posición del sujeto.

Sin duda, la imagen constituye un mediador de la influencia a través de las significaciones elaboradas en la relación del sujeto con la fuente. Y lo constituye porque el sujeto realiza una actividad es- tructurante por la cual llega a representarse la minoria como una categoría más progresista y alternativa en el conjunto del campo social. Esta representación no permite una influencia directa puesto que la identificación puede ser conflictual, pero provoca su impacto sobre la dimensión de los anticonceptivos en la que, como se mostró en la medida de la representación del campo social, el sujeto parece situarse a sí mismo más con respecto a la categoría joven-mujer que con respecto a la fuente minoritaria.

¿Qué ha ocurrido cuando se orienta más la categorización, cuando el sujeto es confrontado a una fuente minoritaria que le puede interpelar más en función de su pertenencia sexual? Respecto a la influencia, se ha visto que cuando la fuente tenía la misma pertenencia sexual que el sujeto se produjo menos influencia que cuando se trataba de una fuente masculina. Este resultado va en contra, pues, de la hipótesis según la cual la comunidad de pertenencias categoriales debería favorecer la influencia de la fuente. ¿Por qué?

A nivel de la representación de la fuente minoritaria se ha visto un sesgo de discriminación intagrupo: se evaluó menos positivamente la fuente intragrupo que a la fuente extragrupo, se la vio más dogmática e incluso feminista y movida por un interés personal.

Si se toman en cuenta los postulados de Maass y cols. (1982), se podria decir que la fuente intragrupo ha obtenido menos influencia que la fuente extragrupo porque ha sido percibida como guiada por más interés personal («self-interest»); y, en efecto, ésa ha sido la tendencia. Se requiere anotar dos precisiones. Por una parte, surge, pues, que el selfinterest no es un sesgo de discriminación frente al extragrupo, sino que parece estar asociado a la categoría sexual femenina, como asociación estereotipada (aborto $=$ affaire de mujeres). Pero, por otra parte, en nuestro caso la atribución del interés personal a la fuente intragrupo ni resulta ser la atribución más pertinente 


\section{Estudios}

(en realidad no se encontró un efecto significativo a nivel estadístico) ni la más apropiada para explicar los resultados de influencia, sin por ello negar tampoco su presencia. En efecto, las dos dimensiones (una evaluación menos positiva y sobre todo el dogmatismo atribuido a la fuente intragrupo por contraste con la fuente extragrupo) en las que se encuentran significativamente diferenciadas estas dos condiciones experimentales, nos obligan a orientarnos hacias otros aspectos.

La evaluación poco positiva y el dogmatismo (dimensión muy próxima a la referida en la literatura como rigidez, cf. Mugny, 1981) han sido ampliamente probados como dos dimensiones que cuando se le atribuyen a la fuente minoritaria anulan (a veces, incluso, producen un efecto "boomerang") la influencia (al menos la directa) de la minoría, por lo que no se plantearía aquí, pues, ningún problema nuevo para explicar la falta de influencia en la condición fuente intragrupo a la que se le han atribuido dichas características. Sin embargo, la cuestión es explicar por qué es la fuente intragrupo la que ha sido juzgada así y no la fuente extragrupo.

Parece claro que los sujetos de la condición intragrupo se han representado al grupo minoritario de jóvenes mujeres como un grupo feminista y le han aplicado asi las características estereotipadas negativas ampliamente difundidas por la ideología dominante. En efecto, cuando al grupo minoritario se le atribuye la característica de feminista y al mismo tiempo se le evalúa negativamente (o menos positivamente que a otras categorias), se le ve también como dogmático y guiado por un interés personal, parecemos estar asistiendo a una forma de "sociologización» (Doise, Deschamps y Mugny, 1980 , p. 102) de la minoría que le impediría justamente ejercer su influencia potencial (merece reseñarse que la dimensión «joven-feminista» también es atribuida en la condición fuente-imagen produciendo un efecto positivo, ahora bien, en esta última condición no se le asociaban caracteristicas negativas). En este caso, todo acercamiento a la posición de la minoría implicaría también la autoatribución de dichas características negativas estereotipadas y como se ha visto los sujetos lo evitan. En otro orden, esta evitación podría significar también la búsqueda de una diferenciación intracategorial. En efecto, entre el sujeto. y la fuente minoritaria intragrupo existe una identidad de hecho, por lo que dicho estereotipo («las feministas») podria aplicarse también a la identidad del sujeto (no olvidemos que también es una mujer). No se puede excluir, pues, que el sujeto tienda en esta condición a preservar su identidad positiva mediante una división intracategorial («Nosotras/ Ellas», Zavalloni, 1971) y que de este modo las características negativas sólo se aplicarian a una parte (pequeña) de las mujeres. Esta interpretación coincide con otros resultados en los que ha sido demostrado también que una fuente categorizada como intragrupo puede inducir un conflicto de identificación y ser por lo tanto más rechazada (Mugny, Kaiser y Papastamou, 1983).

La fuente extragrupo ha obtenido una influencia tanto sobre la dimensión directa como sobre la dimensión indirecta. En la escala de la representación del campo social se vio que no se diferenciaban de la categoría mayoría (categoría con cierta comunidad categorial con la fuente) utilizándola de alguna forma como protección para acercarse a la posición defendida por la minoría. Se vio así en la escala de la representación del campo social que en la dimensión del aborto el propio sujeto no se diferenciaba de la categoria mayoría lo que no ocurria en ninguna otra condición experimental donde el sujeto se situaba más en contra del aborto de lo que situaba la categoria mayoría. Tenemos así que la mayoría puede producir un impacto sobre la posición que el sujeto se atribuye en el campo social respecto a otras categorías sociales.

En este experimento exploratorio, han surgido, pues, dos problemáticas que merecerá seguir analizando: una referida 
al importante papel que parece representar la representación de la fuente minoritaria y que a través de esta representación se puede llegar a percibir y categorizar al grupo minoritario como verdadero portador de una alternativa innovadora (sería éste el efecto del aspecto inductivo sobre el inferencial). Pero se ha visto también que la representación de la minoria puede consistir en la aplicación de una serie de características estereotipadas sobre todo cuando los sujetos juzguen una fuente minoritaria definida categorialmente de antemano (seria éste el efecto del aspecto inferencial sobre el inductivo). Por otra parte, se ha vuelto a demostrar que los procesos de influencia minoritaria, por más afectados que estén, sin embargo, no pueden ser explicados únicamente por un modelo de diferenciación categorial, que llevado a sus últimas consecuencias postularía la discriminación como el único resultado de toda interacción conflictual entre grupos. Aqui hemos visto un efecto más que invierte la lógica, en cierto modo mecanicista, del favoritismo intragrupo. Podemos afirmar ya con fundamento que los procesos de influencia minoritaria quebrantan dicha lógica y que parecen conllevar dinámicas más complejas. A pesar de todo, por el momento nos queda esperar que futuros trabajos vislumbren las dinámicas específicas por las que atraviesan. Tarea que, por medio de este paradigma, que ha resultado eficaz, intentamos llevar a cabo en nuestros trabajos actualmente.

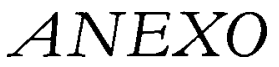

ITEMS DEL CUESTIONARIO DE OPINIONES: El número que encabeza cada item indica el orden en el que se representaba el ítem. La escala de respuesta es dada como ejemplo únicamente en el item 1.

\section{A) DIMENSION DIRECTA: ACTITUD FRENTE AL ABORTO}

1. Una vez legalizado el aborto hay que darle mucha publicidad para que la gente pueda aprovecharse de él.

totalmente en desacuerdo 1234567 totalmente de acuerdo.

2. Hay que informar bien a toda la gente para desculpabilizar a los que recurren al aborto.

3. Todas las clases sociales, ricos y pobres, tienen que tener la misma posibilidad de abortar.

4. Una mujer debe poder abortar sin el consentimiento de su marido.

5. Las menores de edad también deben poder abortar.

6. Hay que legalizar el aborto.

7. El aborto tiene que ser gratuito para todo el mundo.

8. La mujer que ha sido intervenida por aborto debe tener derecho a cierto tiempo pagado de vacaciones.

9. La libertad de abortar constituye otro derecho humano fundamental.

11. Siempre que una mujer ha quedado embarazada por violación hay que poder aplicarle el aborto.

13. El derecho a abortar es una condición indispensable para alcanzar la total dignidad del hombre y de la mujer.

15. El aborto debe ser incluido en el seguro de enfermedad igual que cualquier otro riesgo.

17. El aborto tiene que ser aconsejado a las familias numerosas.

20. El aborto tiene que ser propuesto a las familias con pocos recursos económicos. 


\section{Estudios}

21. Incluso si una persona quisiera abortar, habría que legalizar el aborto.

22. El aborto es una posibilidad para los ricos, debe ser un derecho para la clase obrera.

24. El derecho a abortar es tan importante como el derecho a la huelga.

25. Legalizar el aborto es una exigencia democrática.

B) DIMENSION INDIRECTA: ACTITUD FRENTE A LOS ANTICONCEPTIVOS

10. La legalización del aborto debe ser acompañada de una buena campaña en favor de los anticonceptivos.

12. La utilización de los anticonceptivos no tiene nada que ver con la moral.

14. Se debe informar en los institutos de los anticonceptivos.

16. Hay que realizar una campaña (en la TV) de información en favor de los anticonceptivos.

18. Los anticonceptivos tienen que ser tomados por los dos sexos.

19. Una vez legalizado el aborto el gobierno tiene que realizar una campaña que enfatice la planificación familiar.

23. Los anticonceptivos deben ser pagados por la Seguridad Social.

\section{Referencias}

DOISE, W.: L'articulation psychosociologique et les relations entre groupes. Bruxelas: De Boeck, 1976 (traducción en castellano: Psicologia Social y Relaciones entre grupos. Barcelona: Rol, 1979. 2 vols.).

Doise, W.; Dhschamps, J. C., y Mugny, G.: Psicologia social experimental. Barcelona: Hispano-Europea, 1980.

Lemaine, G.; Lasch, E., y Ricateau, P.: «L'influence sociale et les systèmes d'action: les effets d'attraction et de répulsion dans una expérience de normalisation avec l"callocinétique"». Bulletin de Psychologie, 1971-1972, 25, 482-493.

MaAss, A.; Clark, III R. D., y Haberkorn, G.: «The effects of differential ascribed category membership and norms on minority influence». European Journal of Social Psychology, 1982, 12, 89-104.

MoscovicI, S.: Social influence and social change. Londres: Academic Press, 1976 (traducción en castellano: Psicologia de las minorias activas. Madrid, Morata, 1981).

MoscovicI, S.: "Toward a theory of conversion behavior». En L. Berkowitz (ed.): Advances in experimental social psychology. Nueva York: Academic Press, 1980, vol. 13, 209-239.

Moscovici, S., y Mugny, G.: «Minority influence». En P.B. Paulus (ed.): Basic Group Processes. Nueva York: Springer-Verlag, 1983.

Moscovici, S.; MUGNY, G., y Perez, J. A.: «Les effets pervers du déni (par la majorité) des opinions d'une minorités. En preparación.

MugNy, G.: El poder de las minorias. Barcelona: Rol, 1981. (The posver of minorities. Londres: Academic Press, 1982).

MUGNY, G., y DoISE, W.: “Niveaux d'analyse dans l'étude expérimentale des processus d'influence sociale.) Social Science Information, 1979, 18, 819-876. (Niveles de análisis en el estudio experimental de los procesos de influencia social. Clinica y Análisis Grupal, 1980, 5, 706-745).

Mugny, G.; Kaiser, C., y PAPAstamou, S.: «Influence minoritaire, identification et relations entre groupes: étude expérimentale autour d'une votation». Cabiers de Psychologie Sociale, 1983, 19, 1-30.

MUGNY, G., y PAPASTAMOU, S.: "When rigidity does not fail: individualization and psychologization as resistances to the diffusion of minority innovationsm. European Journal of Social Psychology, 1980, 10, 4361.

MUgny, G., y PAPASTAMOU, S.: "Minority influence and psycho-social identity». European Journal of Social. Psychology, 1982, 12, 379-394.

Mugny, G., y Papastamou, S.: «Les styles de comportement et leur représentation sociale»; En: S. Moscovici. Introduction à la psychologie sociale. Paris, Presses Universitaires de France, en prensa.

Mugny, G.; Rilliet, D., y Papastamou, S.: «Influence minoritaire et identification sociale dans des contextes d'originalité et de déviancem. Revue Suisse de Psychology, 1981, 40, 314-332.

Nemeth C., y WaChTLER, J.: "Creating the perceptions of consistency and confidence: a necessary condition for minority influence». Sociometry, 1974, 37, 529-540. 
Paicheler, G.: «Norms and attitude change I: polarization and styles of behaviour». European Journal of Social Psychology, 1977, 7, 4-14.

Pérez, J. A., y Mugny, G.: "Categorización e influencia minoritaria». Anuario de Psicologia, en prensa.

TAJFEL, H.: «La catégorisation sociale». En S. Moscovici (ed.): Introduction à la psychologie sociale. Paris: Larousse, 1972, vol. 1, 272-300.

TAJFEL, H. (Ed.): Differentiation between social groups: studies in social psychology. Londres: Academic Press, 1978.

TURNER, J. C.: “Towards a cognitive redefinition of the social group». Cabiers de Psychologie Cognitive, 1981, 1, 93-118.

VANBESELAERE, N.: “Mere exposure search for one explanation». En: W. Doise y S. Moscovici (eds.): Current issues in european social psychology. Cambridge, Cambridge University Press-L.E.P.S., 1983, vol. 1, 239-278.

ZAVALLONI, M.: "Cognitive processes and social identity through focused introspection». European Journal of Social Psychology, 1971, 2, 235-260. 